\title{
The Role of Cation Intermixing, Interfacial Chemistry, and Oxygen Deficiency in Understanding the Properties of the $\mathrm{LaFeO}_{3} / \mathrm{SrTiO}_{3}(100)$ Interface
}

\author{
R. Colby ${ }^{1}$, K.H.L. Zhang ${ }^{2}$, A. Genc ${ }^{3}$, L. Pullan ${ }^{3}$, B. Kabius ${ }^{1}$, S.A. Chambers ${ }^{2}$ \\ ${ }^{1}$ Environmental Molecular Sciences Laboratory, Pacific Northwest National Laboratory, Richland, WA, \\ 99354 \\ ${ }^{2}$ Fundamental and Computational Sciences Directorate, Pacific Northwest National Laboratory, \\ Richland, WA, 99354 \\ ${ }^{3}$ FEI Company, Hillsboro, OR 97124, USA
}

The properties of oxide perovskites vary broadly for different cations and stoichiometries, and interfaces between dissimilar oxides can have entirely novel characteristics not seen in the bulk of either material. The anomalous conducting interface between $\mathrm{LaAlO}_{3}$ and $\mathrm{SrTiO}_{3}(001)$ has received the most attention, and numerous hypothetical explanations have been posited related to resolution of the polar discontinuity at the interface, including cation inter-diffusion, interfacially-reduced B-site cations, local oxygen deficiency, and various combinations thereof [1-3]. The growing body of research indicates a diverse interplay of these effects in various polar/non-polar perovskite oxide interfaces-possibly specific to each materials system - and a coherent approach to predicting the properties of such interfaces remains elusive.

In past work, it has been shown that the $\mathrm{LaCrO}_{3} / \mathrm{SrTiO}_{3}(001)$ interface, grown by molecular beam epitaxy (MBE), was insulating despite having uneven inter-diffusion of cations and locally reduced $\mathrm{Ti}$ and $\mathrm{Cr}$ near the interface [4]. Analysis of the net charge suggested that the interface must have been oxygen deficient, on the LCO side. Here, we build upon that work by examining the similar interface between polar $\mathrm{LaFeO}_{3}$ (LFO) and non-polar $\mathrm{SrTiO}_{3}$ (STO), down to atomic resolution, using aberration-corrected scanning transmission electron microscopy (STEM), electron energy loss spectroscopy (EELS), and an FEI Titan TEM with ChemiSTEM technology for high sensitivity x-ray energy dispersive spectroscopy (EDS).

As-grown LFO/STO(001) interfaces are insulating at room temperature. High resolution TEM and STEM indicate high quality epitaxial growth and rhombohedral LFO, as shown in Fig. 1(a). High angle annular dark field (HAADF)-STEM, EELS, and EDS maps all indicate significant inter-diffusion of cations across the original interface, spanning several nanometers. The extent of diffusion is significantly larger for $\mathrm{Sr}$ than $\mathrm{La}$, followed by $\mathrm{Fe}$ and $\mathrm{Ti}$, as can be seen in Fig. 1(b). By examination of the position and shape of the Fe- $\mathrm{L}_{3}$ edge, using EELS, it is found that the bulk of the LFO thin film is predominantly $\mathrm{Fe}^{3}+$, with a fraction of $\mathrm{Fe}^{2}+$ indicating a slight oxygen deficiency. Comparison of the $\mathrm{O}$ $\mathrm{K}$-edge in the film and substrate using EELS is consistent with a slightly oxygen deficient as-grown film, as is the EDS analysis. The $\mathrm{Fe}^{2}+: \mathrm{Fe}^{3}+$ ratio increases approaching the interface, as shown in Fig. 1(c). The Fe reduction corresponds to a local O-deficiency on the LFO side of the interface, as observed in the EDS maps. Unusually for such systems, the $\mathrm{Ti}$ L-edge is consistent with $\mathrm{Ti}^{4+}$ throughout, having no indication of the interfacial Ti-reduction that is the hallmark of the electronic reconstruction description of similarly Ti-terminated $\mathrm{LaAlO}_{3} / \mathrm{SrTiO}_{3}(001)$.

A number of groups have found that as-grown oxygen-deficient perovskite films can sometimes be oxidized at temperatures well below the growth temperature in ambient pressure oxygen or ozone [5]. 
After annealing the $\mathrm{LFO} / \mathrm{STO}(001)$ in air at $350^{\circ} \mathrm{C}$ for several hours ( 8 hours), the $\mathrm{Fe}^{2+} \mathrm{Fe}^{-\mathrm{L}_{3}}$ feature was found to be absent throughout the film and interface. The inter-diffusion of the four cations was found to be nominally unchanged. However, both the film and interface remained insulating at room temperature. These results suggest that the emergence of conductivity at polar/non-polar perovskite interfaces is not a simple function of only the local oxygen deficiency, interfacial cation reduction, or uneven cation inter-diffusion.

\section{References}

[1] S. A. Chambers, Surf. Sci. 605 (2011) 1133.

[2] A. Ohtomo, H.Y. Hwang, Nature 427, (2004) 6973.

[3] V. Vonk, et al., Phys. Rev. B 85 (2012) 045401.

[4] R. Colby, et al., Phys Rev B 88, (2013) 155325.

[5] Y. Xie, et al., Advanced Materials, DOI: 10.1002/adma.201304374, (2013)

[6] This work was supported by the U.S. Department of Energy, Office of Science, Division of Materials Sciences and Engineering under Award \#10122, Division of Chemical Sciences under Award \#48526, and the EMSL William Wiley Postdoctoral Fellowship program. The work was performed in the Environmental Molecular Sciences Laboratory, a national science user facility sponsored by the Department of Energy's Office of Biological and Environmental Research and located at Pacific Northwest National Laboratory.
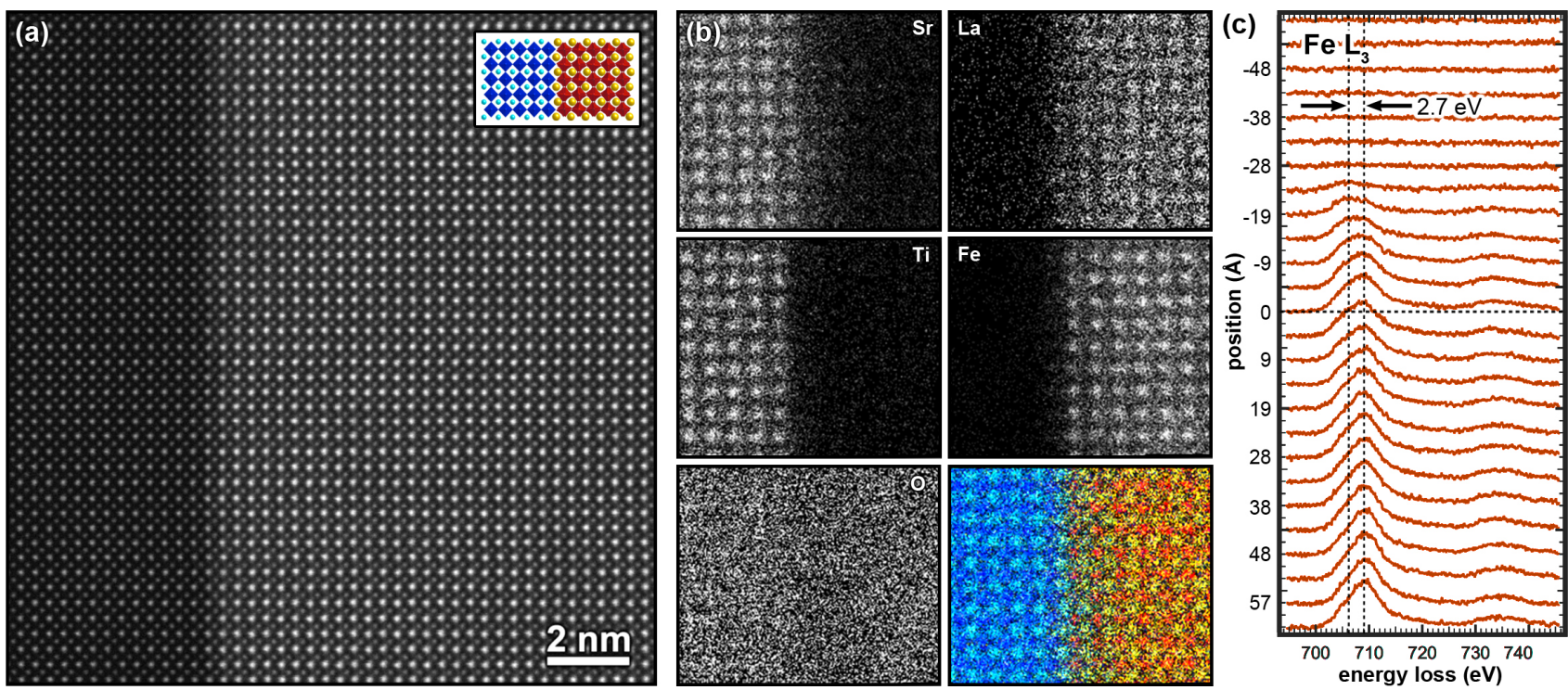

Figure 1: (a) HAADF-STEM image of a typical region of the LFO/STO(001) interface and inset model of an ideal interface. (b) STEM-EDS maps of the Sr, La, Ti, Fe, O, and a colored overlay of the cations with $\mathrm{Sr}$ (cyan), La (yellow), Ti (blue), and Fe (red). The Sr evidences the greatest extent of out-diffusion, Ti the least. There is a slight O-deficiency in the near-interface LFO. (c) EELS of the Fe L-edge in the as-grown films indicates an increasing ratio of $\mathrm{Fe}^{2+}: \mathrm{Fe}^{3+}$ approaching and passing through the interface (the position is positive in the growth direction, i.e. towards the LFO surface). The spectra have been background subtracted, and adjusted for absolute energy based on the concurrently acquired positions of the zero-loss peak. The $\mathrm{Fe}^{2+}: \mathrm{Fe}^{3+}$ ratio is derived by fitting two Gaussians separated by 2.7 $\mathrm{eV}$, consistent with literature differences for $\mathrm{Fe}^{3+}$ and $\mathrm{Fe}^{2+}$ in oxides. 